\title{
Efecto de reprocesos de fundición e inyección en la microestructura y las propiedades mecánicas de aleaciones Zamak
}

\section{Effect of melting and injection reprocesses on the microstructure and mechanical properties of Zamak alloys}

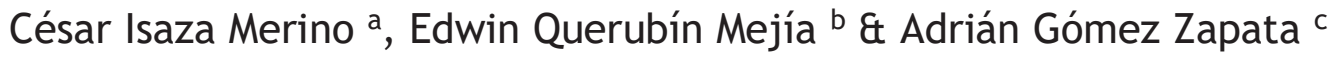 \\ a Docente, Institución Universitaria Pascual Bravo, Grupo GIIEN, Medellín, Colombia, c.isaza2059@pascualbravo.edu.co \\ ${ }^{b}$ Institución Universitaria Pascual Bravo, Medellín, Colombia. e.querubin1503@pascualbravo.edu.co \\ c Docente, Institución Universitaria Pascual Bravo, Grupo GIIEN, Medellín, Colombia, adrian.gomez@pascualbravo.edu.co
}

Recibido: octubre 6, 2019. Aceptado: noviembre 20, 2019.

\section{Resumen}

En este trabajo se estudió el efecto que tiene el proceso de fundición e inyección en la microestructura y las propiedades mecánicas de una aleación metálica no ferrosa base zinc-aluminio (Zn-Al) conocida como Zamak. Los análisis se realizaron sobre muestras obtenidas de un lingote en estado de entrega, es decir, tal como llega de los proveedores al proceso productivo de fundición e inyección de piezas de bisutería; y sobre muestras extraídas de un lingote fabricado con una mezcla de material nuevo (70\% de la muestra inicial) y material reciclado $(30 \%)$ proveniente de los rebordes y excedentes de los procesos de fundición e inyección de las mismas piezas de bisutería. Mediante ensayos de metalografía se comprobó que, tanto las muestras iniciales como las secundarias, contienen microestructuras formadas por una fase de solución sólida y una fase eutéctica en diferentes proporciones. Además, se observó que el crecimiento de precipitados en la muestra secundaria, indica que hubo un refinamiento o redistribución de la estructura conformada por granos de fase sólida, los cuales se tornaron en formas más redondeadas y abundantes en la muestra. Por medio de difracción de rayos $\mathrm{X}$, no se evidenció la formación de nuevas fases en la muestra secundaria. Para la cuantificación de las propiedades mecánicas se recurrió a ensayos de nanoindentación, macrodureza y ensayos de tensión, cuyos resultados mostraron un ligero cambio en las propiedades mecánicas, que para el proceso productivo de fundición y conformado volumétrico por inyección, no representan una contribución muy significativa en piezas con las mismas especificaciones fabricadas con ambos tipos de materiales. Por tal razón, el reprocesado de material en el proceso productivo de muestras para bisutería es una alternativa importante para el aumento de la productividad y la reducción de costos en la manufactura por fundición e inyección de este tipo de piezas. Palabras Clave: Zamak, microestructura, nano-indentación, propiedades mecánicas, reprocesos.

\begin{abstract}
The effect of melting and injection processes on microstructural and mechanical properties of zinc-aluminum based (Zn-Al) non-ferrous alloys (Zamak) were studied. The analyses were carried out on samples from an ingot coming from the raw material used in the costume jewelry manufacturing process. The second one was extracted from an ingot cast with a mix of new material $(70 \%$ of the primary sample) and recycled material ( $30 \%)$ derived from the excess of melted and injected samples on molds in this manufacturing process. Tests detected both a solid solution and eutectic phases in different amounts. The growth of the precipitates surrounding the solid phase was more evident in the secondary sample than the first one. The growth points out to refinement or redistribution of the structure composed by solid phase grains, more rounded, and abundant for the second sample. No secondary or new phases were detected through the DRX technique for the second sample. The assessment of mechanical properties was carried out using nano-indentation, macro-hardness, and strain tests. The results show a slight change in the mechanical properties. Nevertheless, for the melting and injection volumetric shape processes, the influence is negligible for shapes with the same specifications produced with both kinds of materials. Therefore, the material recycling process is an essential option for increasing the productive capacity and vital alternative to reduce the cost in the costume jewelry manufacturing process by melting and injection.
\end{abstract}

Keywords: Zamak, microstructure, nano indentation, mechanical properties, reprocesses.

\section{INTRODUCCIÓN}

Uno de los principales retos en la industria metal-mecánica es encontrar un punto óptimo entre la calidad y los precios [1]. Es por ello por lo que siempre se han buscado alternativas para poder obtener piezas de buena calidad, con especificaciones mecánicas mejoradas y al menor costo posible. En esta industria, más

\section{Citar como:}

C. Isaza, E. Querubín, A. Gómez. "Efecto de reprocesos de fundición e inyección en la microestructura y las propiedades mecánicas de aleaciones Zamak". Revista CINTEX, Vol. 24(1), pp. 33-40. 2019. 
específicamente en la relacionada con lo ornamental o de bisutería, se busca encontrar y probar diversos materiales que cumplan con requerimientos y especificaciones tales como, buena calidad y propiedades mecánicas a menor precio [2], [3].

Dentro de los materiales utilizados para la fabricación de elementos de bisutería para prendas de vestir se encuentran aleaciones tales como el latón; el cual es una aleación de cobre y zinc, donde éste último puede variar entre un 0 y $50 \%$ y dependiendo de esta variación pueden recibir diferentes nombres: bronce $(10 \%)$, latón rojo (15\%), latón amarillo (34-37\%), y latones modificados cuando contiene otros elementos de aleación; la alpaca; aleación de cobre, zinc y níquel; y la bittania; aleación de estaño $(93 \%)$, cobre $(2 \%)$, y antimonio $(5 \%)$ [4], [5]. En la actualidad existe un material a base de zinc, que además de cumplir con los requerimientos de buena calidad a un bajo costo, posee buenas propiedades mecánicas, buena resistencia a la corrosión, y proporciona muy buenos acabados y versatilidad en los diseños de formas y texturas, lo que lo hace muy atractivo en el área de la bisutería; dichos materiales o aleaciones son conocidos como Zamak [6]. Igualmente, otra de las ventajas de las aleaciones Zamak, es que no contienen elementos de aleación o contaminantes considerados tóxicos como el plomo, el cadmio y el antimonio, o elementos alergénicos como el níquel y el cobalto, que pueden causar malestares y fuertes irritaciones en el cuerpo.

Los materiales Zamak más comunes son los referenciados como Zamak 2, Zamak 3, Zamak 5 y Zamak 7 , cuya diferencia radica en el porcentaje de cobre que poseen [7]. Las aleaciones Zamak son materiales metálicos no ferrosos a base de zinc $(\mathrm{Zn})$ y aluminio ( $\mathrm{Al})$ en combinación con otros elementos tales como magnesio $(\mathrm{Mg})$ y cobre $(\mathrm{Cu})$. De estas, las más utilizadas en la industria de la bisutería son el Zamak 3 y Zamak 5, que corresponden a las aleaciones normalizadas SAE 903 y SAE 925, respectivamente. Para la transformación de las aleaciones Zamak, los procesos de manufactura más utilizados son los procesos de fusión, solidificación y de conformado volumétrico como la inyección.

En los procesos de fundición e inyección de piezas de Zamak, la opción del reciclaje de los excedentes o rebordes que quedan después de dichos procesos, es siempre una alternativa que se ha contemplado para reducir los costos de producción de piezas de bisutería [3], [8], ya que esto permite la fabricación de formas; dependiendo de las características y propiedades requeridas en estas; con lingotes conformados por mezclas hasta con un $80 \%$ de material reciclado (información suministrada por la industria que facilitó los materiales para la realización de este trabajo). Si bien se han reportado varios estudios con relación a los efectos de la microestructura sobre el desempeño mecánico y a la fricción de algunas aleaciones tipo Zamak [9]-[11], aún no se encuentran reportados efectos de reprocesos de fundición e inyección de Zamak reciclado sobre la microestructura y las propiedades mecánicas de éste.

El presente estudio muestra los resultados de la caracterización microestructural y las propiedades mecánicas realizadas a muestras de una aleación tipo Zamak obtenidas de un lingote nuevo (muestra Z1) y a muestras obtenidas de un lingote conformado por material nuevo (70 \% Z1) más material reciclado $(30 \%)$ proveniente de excedentes de los procesos de pulido y de rebordes después del proceso de fundición e inyección de piezas en una planta para fabricación de accesorios y elementos de bisutería para prendas de vestir, las cuales se etiquetaron Z2. Para la evaluación de las diferentes propiedades, se utilizaron técnicas de caracterización como metalografía, espectroscopía de emisión óptica, microscopia óptica, ensayos de nanoindentación, microdureza y ensayos de tensión [12], [13].

\section{PROCEDIMIENTO EXPERIMENTAL}

El análisis microestructural se realizó sobre una probeta extraída de cada uno de los lingotes Z1 y Z2. Para las observaciones al microscopio, las probetas Z1 y Z2 fueron preparadas con base en los lineamientos descritos en la norma ASTM E-3 "Preparación de muestras metalográficas" [14]. Las micrografías fueron adquiridas a través de un microscopio metalográfico marca Olympus referencia PME-3, el cual tiene adaptada una cámara digital marca Nikon serie Lv 100 y software para análisis de imagen. Estas micrografías fueron tomadas a diferentes magnificaciones o aumentos en diferentes regiones a lo largo y ancho de las muestras con el fin de identificar la morfología y la distribución de las fases presentes en las aleaciones.

La composición química elemental se hizo sobre tres muestras de cada uno de los materiales Z1 y Z2, la cual fue determinada a través de la técnica de Espectroscopía de Emisión Óptica (OES, por sus siglas en inglés), utilizando para ello un equipo marca Shimadzu referencia OES-5500 con el fin de verificar e identificar las cantidades porcentuales y variaciones composicionales de los elementos presentes en las aleaciones que pudieran afectar el comportamiento microestructural y mecánico de éstas. Para corroborar las fases presentes en las muestras Z1 y Z2, se realizó, por medio de la técnica de Difracción de Rayos X (DRX), un análisis estructural de éstas utilizando para ello un difractómetro de rayos X marca Bruker referencia D8 Advance Eco.

La estimación de las propiedades mecánicas se realizó por medio de diferentes técnicas de caracterización, entre las cuales están ensayos de macro-dureza, nanoindentación y ensayos de tensión. Los ensayos de dureza se realizaron en un durómetro marca Wolpert referencia Dia Testor 2-Rc de acuerdo al procedimiento 
descrito en la norma ASTM E-92 "Método de ensayo estandarizado de dureza Vickers y Knoop en materiales metálicos" [15]. Los ensayos de nanoindentación se realizaron en un nanoindentador marca lbis Authority con una punta tipo Berkovich, con el fin de identificar el comportamiento mecánico (dureza y módulo de elasticidad) de las fases presentes en las aleaciones Z1 y Z2. Para los ensayos de tensión, se obtuvieron tres (3) probetas cilíndricas de cada uno de los lingotes Z1 y Z2, las cuales fueron maquinadas mediante torno convencional de acuerdo a dimensiones y especificaciones descritas en la norma ASTM E-8 "Ensayo de tracción de materiales metálicos" [16]. La

Figura 1 corresponde a una de las muestras obtenidas por el proceso de mecanizado. Los ensayos de tracción de las probetas se realizaron en una máquina universal marca Shimadzu referencia GX con capacidad para aplicar carga hasta 30 toneladas. De las gráficas resultantes de los ensayos de tracción se leyeron directamente los valores de propiedades como esfuerzo de cedencia, resistencia última y ductilidad.

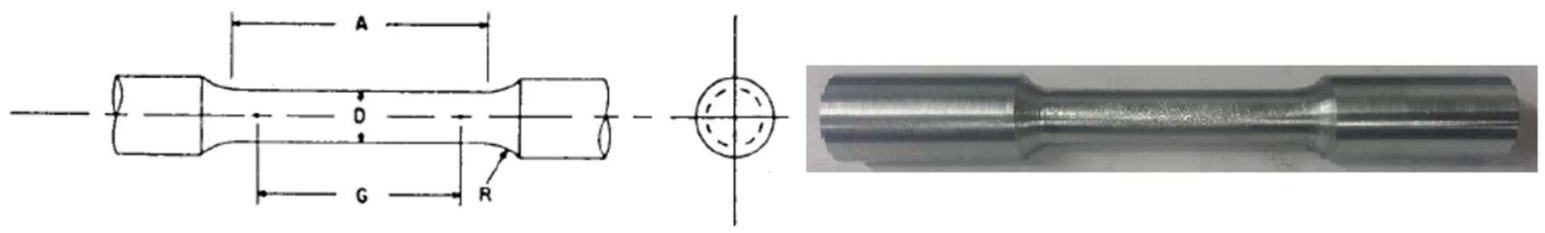

Longitud de Prueba G: 50 mm, Diámetro D: 12.5 mm, Radio de Curvatura R:10 mm, longitud Reducida A: 56 mm

Figura 1. Probeta de tracción obtenida de lingote de Zamak y maquinada por medio de torno convencional de acuerdo con dimensiones para el espécimen 1 establecidas en la norma ASTM E-8.

\section{RESULTADOS Y DISCUSIÓN}

\subsection{Caracterización microestructural}

Las Figuras 2 y 3 corresponden a la microestructura observada en la muestra del material en estado de entrega $Z 1$ y al material reprocesado Z2, respectivamente; ambas microestructuras están compuestas por granos de fase $\alpha$ perteneciente al aluminio (Al) y fase $\beta$ perteneciente al zinc $(\mathrm{Zn})$. En las imágenes se pueden observar detalles o magnificaciones sobre un punto específico de las muestras, que permiten evidenciar con mayor detalle las dendritas formadas durante el proceso de solidificación lento que forma una solución sólida a y una configuración de fases eutéctica $(\alpha+\beta)$, es decir, láminas intercaladas de fase $\alpha$ y fase $\beta$. En la Figura 3 se puede ver un cambio microestructural debido al refinamiento de grano sufrido después del reproceso.

La fase clara de la transformación eutéctica es la solución sólida $\alpha$. La fase gris de la transformación eutéctica es la solución sólida $\beta$. Se puede observar que el crecimiento de precipitados de fase $\beta$ alrededor de la fase $\alpha$ es más notorio en la muestra Z2, lo que indica que hubo un refinamiento o redistribución de la estructura y, por ende, de los granos que conforman la fase $\alpha$, conllevando con ello a granos de fase $\beta$ más redondeados y abundantes en la muestra Z2. También se puede observar que la forma de crecimiento de las dendritas de la muestra Z1 es parecida al de una ramificación de una planta, lo que conlleva a granos con formas alargadas. En la muestra Z2 se observa una microestructura compuesta por las mismas fases que conforman la muestra Z1, pero a diferencia de ésta, se puede ver que la fase a se presenta en mayor proporción a la de la muestra Z1, lo cual se hace más visible a medida que se hace una magnificación de la zona observada. Igualmente se puede observar, que la fase a de la muestra Z2 se presenta en forma de granos más redondeados; como ya se había mencionado, esto fue debido probablemente al proceso de refundido y reinyectado que sufre la muestra $Z 2$, además de la combinación en cierta cantidad de material reciclado con parte de muestra Z1.
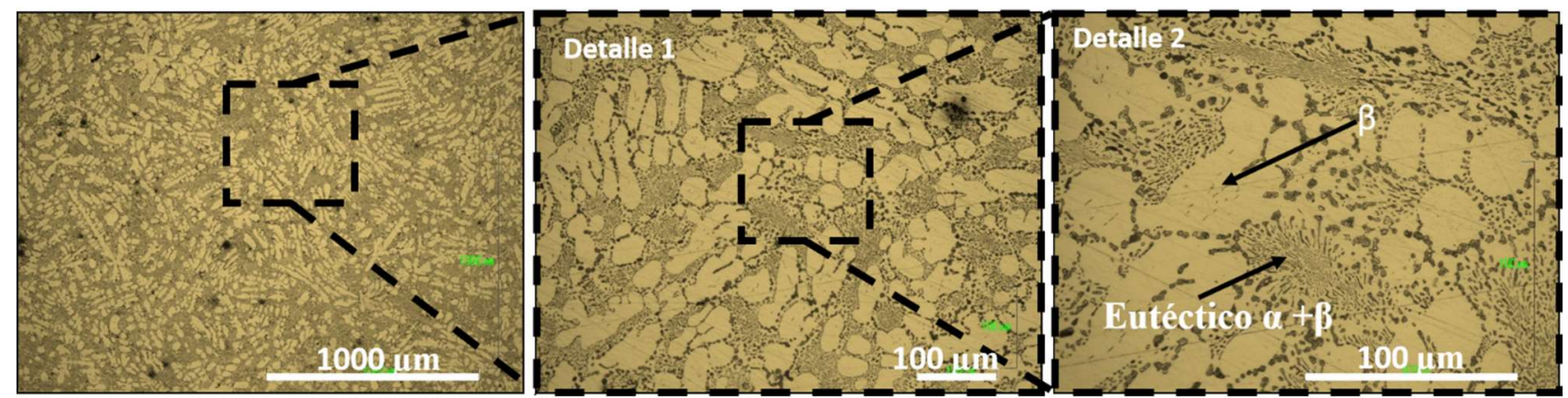

Figura 2. Microestructura compuesta de fase $\bar{\alpha}(\overline{\mathrm{Al}})$ y fase $\beta(\mathrm{Zn})$ correspondiente a las muestras $\mathrm{Z1}$. 

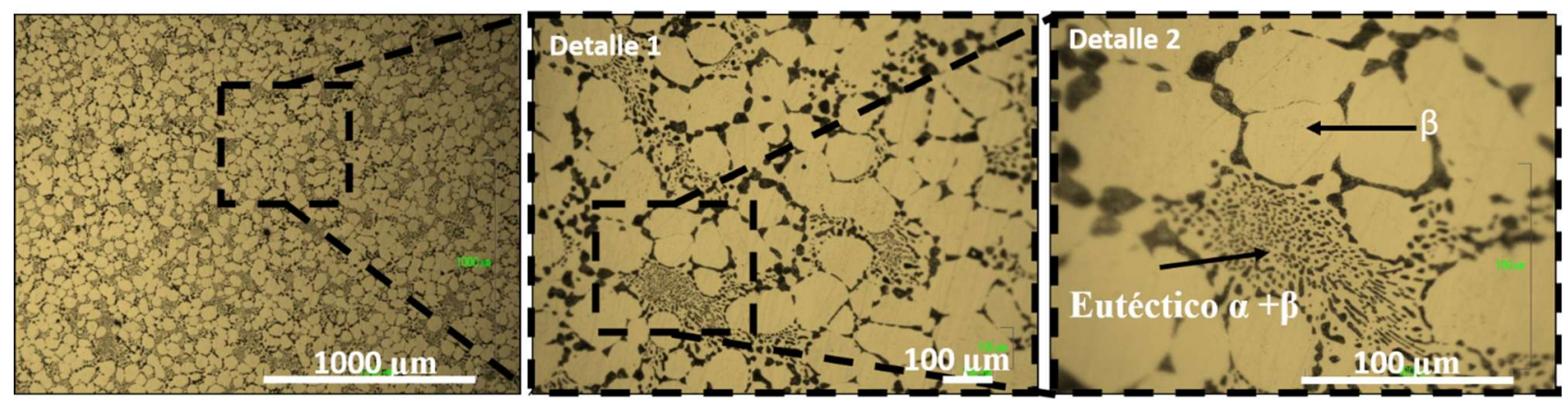

Figura 3. Microestructura compuesta de fase $\alpha(\mathrm{Al})$ y fase $\beta(\mathrm{Zn})$ correspondiente a las muestras $Z 2$.

\subsection{Caracterización composicional y de fases}

\subsubsection{Espectroscopía de Emisión Óptica (OES)}

Se realizó un análisis de composición química en tres muestras de cada material, con el fin verificar e identificar las cantidades porcentuales y las variaciones composicionales de los elementos presentes en las aleaciones que pudieran afectar el comportamiento microestructural y mecánico de estos materiales. La Tabla 1. corresponde a los resultados del análisis composicional de las dos muestras, cuyos resultados evidencian que el porcentaje en peso del zinc disminuye aproximadamente $\approx 1 \%$ y hay un leve aumento del porcentaje en peso del aluminio de $\approx 5.6 \%$ y del cobre $\approx 40 \%$ para la muestra Z2 con relación a Z1, cuyos elementos le proporcionan un refinamiento del grano, mayor resistencia mecánica y mejor resistencia a la corrosión, respectivamente, a la muestra reprocesada (Z2), y un cambio en sus cantidades puede influir en dichas propiedades. Estas variaciones composicionales pueden deberse a que el material reprocesado que se mezcla con material en estado de entrega Z1, puede provenir de procesos en los cuales se pudo haber trabajado con otros tipos de materiales Zamak con diferentes composiciones químicas. El cambio microestructural generado por el aumento o disminución en las cantidades de los elementos constituyentes de la aleación se pudo apreciar en las imágenes de la Figura 2 y 3.

Tabla 1. Resultados del análisis composicional; en \% en peso; para las muestras Z1 y Z2

\begin{tabular}{ccc} 
Elemento & $\begin{array}{c}\text { Porcentaje en } \\
\text { Peso - Z1 }\end{array}$ & $\begin{array}{c}\text { Porcentaje en } \\
\text { Peso - Z2 }\end{array}$ \\
\hline $\mathrm{Zn}$ & 93,61 & 92,99 \\
$\mathrm{Al}$ & 4,73 & 5,01 \\
$\mathrm{Cu}$ & 0,77 & 1,28 \\
$\mathrm{Mg}$ & 0,39 & 0,36 \\
$\mathrm{Si}$ & 0,06 & $\ldots$ \\
$\mathrm{Fe}$ & 0,03 & 0,02 \\
$\mathrm{Ni}$ & 0,01 & 0,02 \\
$\mathrm{Cr}$ & 0,01 & 0,01 \\
$\mathrm{~Pb}$ & 0,01 & $\ldots$ \\
$\mathrm{Ca}$ & 0,01 & $\ldots$ \\
\hline
\end{tabular}

\subsubsection{Difracción de Rayos X (DRX)}

Del diagrama de fases para aleaciones de $\mathrm{Zn}-\mathrm{Al}$ [7] que se muestra en la Figura 4.a., se sabe que esta aleación termina su solidificación en una eutéctica ternaria que se presenta alrededor de $382^{\circ} \mathrm{C}$ : líquido para dar $(\alpha+\beta+\varepsilon)$; siendo $\beta$ la solución sólida rica en Al que cristaliza en el cúbico centrado en las caras; $\alpha$ la 
solución rica en $\mathrm{Zn}$ que cristaliza en el sistema hexagonal compacto; y $\varepsilon$, el compuesto inter-metálico rico en Zn, que cristaliza en el sistema hexagonal. La composición de la eutéctica ternaria es $7.05 \%$ de Al, $3.85 \%$ de Cu y $89.10 \%$ de $\mathrm{Zn}$; y las fases en equilibrio son líquido (7\% de $\mathrm{Al}, 3.9 \%$ de $\mathrm{Cu}$ y resto $\mathrm{Zn}$ ), fase $\alpha(1.3 \% \mathrm{Al}, 2.9 \%$ $\mathrm{Cu})$; fase $\beta(19.5 \% \mathrm{Al}, 1.8 \% \mathrm{Cu}$, resto $\mathrm{Zn})$ y la fase $\varepsilon(1.4 \% \mathrm{Al}, 15.2 \% \mathrm{Cu}$ y el resto $\mathrm{Zn})$. Como se observa, la composición química y la fase en equilibrio a $(89.1 \% \mathrm{Zn})$, presentaron un comportamiento acorde al reportado en el diagrama de fases, con ligeras variaciones de $\approx 5.1 \mathrm{y} \approx 4.4 \%$, para las muestras $Z 1$ y $Z 2$, respectivamente, con relación a la composición química reportada por la técnica OES.

Por otro lado, el análisis de DRX realizado sobre las muestras en estado de entrega (Z1) y reprocesada (Z2) (Figura 4.b.) evidenció, dentro de las limitaciones de la técnica, que no hubo formación de nuevas fases cristalinas, a pesar del aumento mostrado por el aluminio y el cobre reportados en la composición química. Sin embargo, esta afirmación necesita ser validada por medio de una técnica más especializada, como la Difracción de Rayos X por Luz Sincrotrón, ya que la difracción convencional; como la utilizada para este trabajo; sólo está en capacidad de detectar fases que se encuentren en una concentración por encima del $5 \%$ en peso en un material, que para nuestro caso, está muy cercano al de la eutéctica ternaria, donde las fases ricas en aluminio $(\mathrm{Al})$ y cobre $(\mathrm{Cu})$ no superan este valor. Esto corrobora tanto la información reportada por la metalografía, como la de composición química, ya que la única fase detectada por DRX fue la fase rica en Zinc, para la cual se detectaron los planos de difracción principales $\left(\begin{array}{lll}0 & 1 & 2\end{array}\right),\left(\begin{array}{lll}1 & 0 & 4\end{array}\right)$ y $\left(\begin{array}{lll}0 & 1 & 5\end{array}\right)$, tanto para la muestra Z1 como para Z2. Igualmente, se puede observar en la Figura 4.b. que la única diferencia en los difractogramas es la altura de los picos, esto debido a la diferencia en el contenido del zinc (Zn) en las muestras.
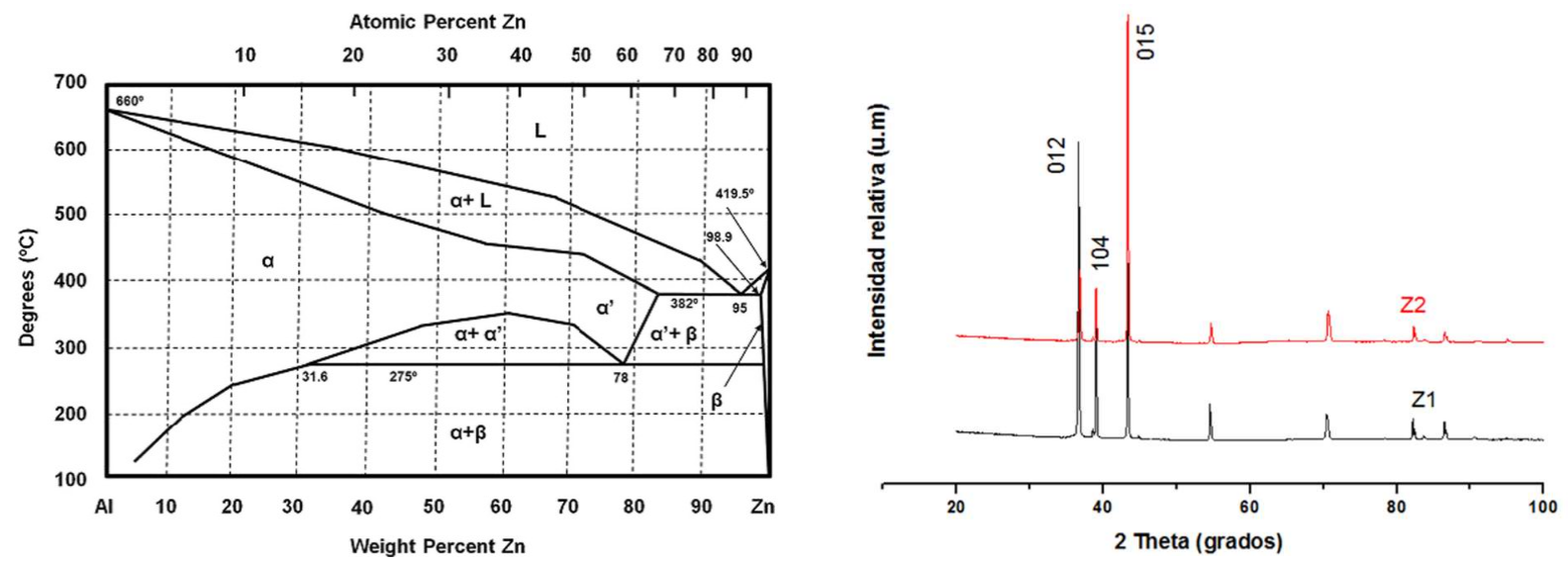

b)

Figura 4. a) diagrama de fases de Zn - Al y b) Ensayos de difracción de rayos X para las muestras Z1 y Z2.

\subsection{Caracterización mecánica}

\subsubsection{Resultados de nanocaracterización}

Para determinar las propiedades mecánicas de las muestras Z1 y Z2, se utilizaron técnicas de caracterización tales como nanoindentación, macrodureza y ensayos de tensión. En la Figura 5.a se pueden ver los ensayos de nanoindentación y los resultados de módulo de elasticidad (Figura 5.b) y de dureza (Figura 5.c) para la muestra Z1. Se evidenció que tanto el módulo de elasticidad como la dureza variaron con respecto a la fase medida, es decir, el módulo de elasticidad para la fase $(\alpha+\beta)$ fue más bajo (100 GPa) que para la fase $\beta(120 \mathrm{GPa})$. La dureza tuvo el mismo comportamiento, es decir, fue más dura la fase $\beta$, con una dureza promedio de $1.3 \mathrm{GPa}$, que la fase eutéctica $(\alpha+\beta)$ con una dureza promedio de $0.9 \mathrm{GPa}$. Este resultado evidencia que la fase eutéctica que se forma con las fases $\alpha$ y $\beta$ ricas en aluminio y en zinc, respectivamente, proporcionan una disminución de la dureza y, por ende, una mayor capacidad de deformación plástica durante los procesos de conformado volumétrico.

El mismo análisis fue realizado para la muestra Z2, como se muestra en la Figura 6 . De los resultados obtenidos se evidenció que el comportamiento fue similar, es decir, la fase eutéctica $(\alpha+\beta)$ presentó propiedades inferiores de módulo de elasticidad y de dureza con respecto a la fase $\beta$ presente en la aleación. Sin embargo, se puedo observar que las propiedades mecánicas fueron ligeramente inferiores en comparación con las muestras del material en estado de entrega (Z1). Esto pudo deberse a la distribución de la fase eutéctica que se disolvió hacia los límites de grano, permitiendo al mismo tiempo una formación equiaxial de la fase $\beta$. 

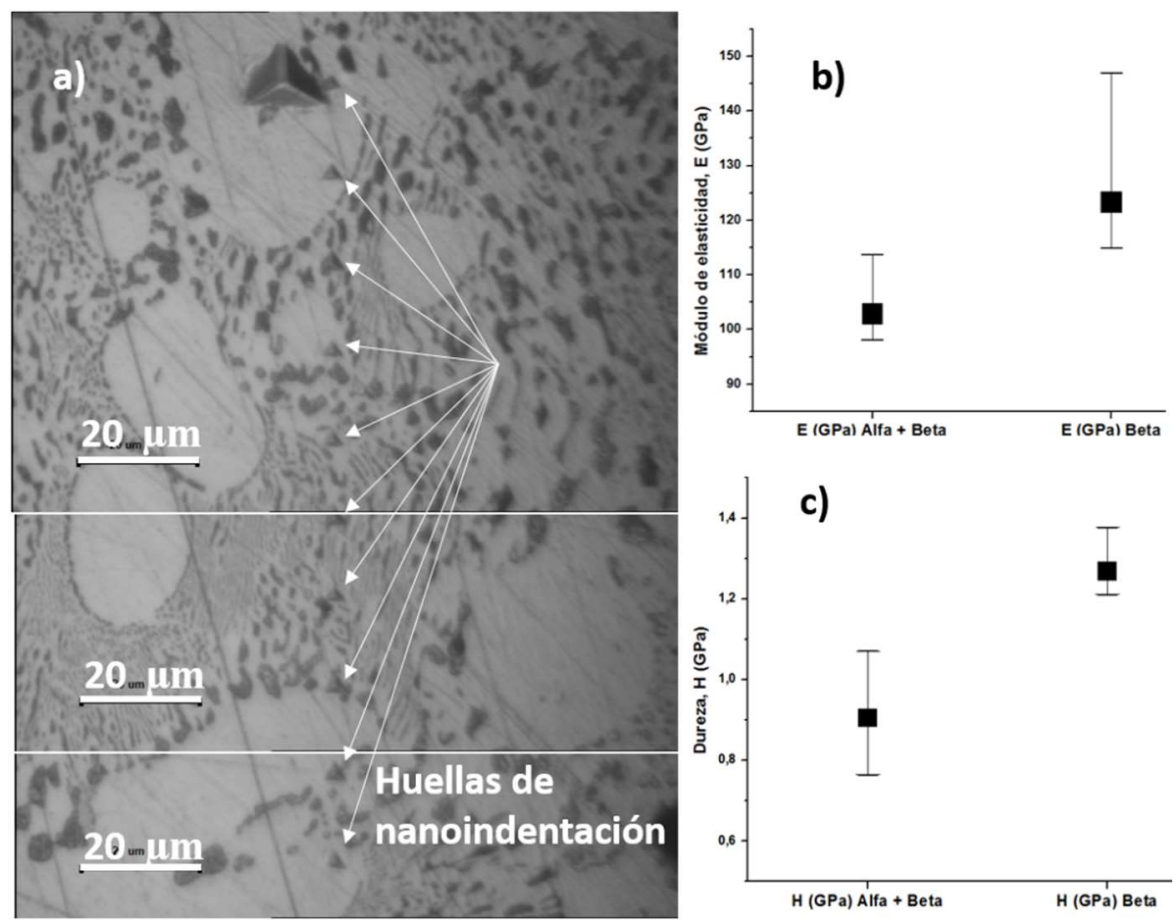

Figura 5. Resultados de nanoindentación para la muestra Z1, a) imágenes de las huellas de nanoindentación, b) módulo de elasticidad y c) dureza.
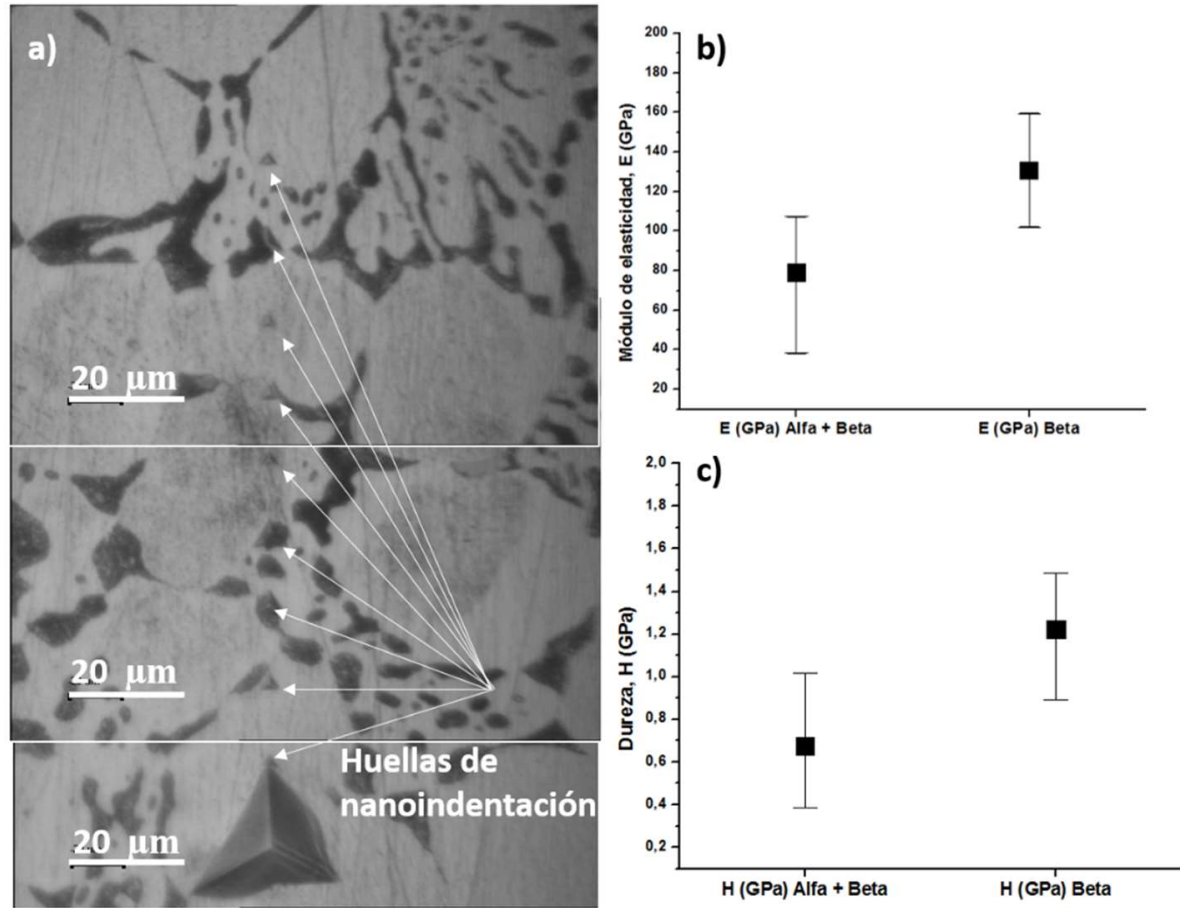

Figura 6. Resultados de nanoindentación para la muestra Z2, a) imágenes de las huellas de nanoindentación, b) módulo de elasticidad y c) dureza.

\subsubsection{Resultados de macrocaracterización}

Los ensayos de caracterización mecánica a nivel volumétrico son mostrados en las imágenes de la Figura 7 , donde los resultados de macrodureza se muestran en la Figura 7.a y los ensayos de tensión en la Figura 7.b. En la muestra Z2 se evidenció que la dureza tuvo un aumento, pasando de $85 \pm 7$ Vickers en las muestras en estado de entrega (Z1) a $96 \pm 4$ Vickers en las muestras reprocesadas (Z2). Esto pudo deberse a que el proceso de recristalización obtenido por la distribución de la fase $(\alpha+\beta)$ no permitió una libre deformación del grano, debido a que el movimiento de dislocaciones se vio restringido por el límite del grano equiaxial formado. Sin 
embargo, la diferencia de dureza no fue estadísticamente significativa como para que haya una influencia directa sobre el proceso de conformado volumétrico.

De igual forma, los resultados de tensión (Figura 7.b) mostraron un comportamiento muy similar entre las muestras en estado de entrega Z1 (256MPa) y las muestras reprocesadas Z2 (269MPa). Se observó que la muestra Z2 (reprocesada) tuvo una resistencia máxima a la tensión ligeramente superior en comparación con la muestra en su estado de entrega (Z1). También se evidenció un ligero aumento de la tenacidad del material Z2; es decir, que la capacidad del material de deformarse plásticamente aumentó (ver Figura 7.b). Aunque este resultado va en contravía con la microestructura de la muestra Z2, la cual evidenció algo de recristalización, esto pudo deberse a que la disolución de la fase eutéctica (fase blanda) hacia los límites de grano permitió el movimiento entre granos, lo que pudo ser limitado pero suficiente para generar este cambio en la ductilidad del material. Además, de la cuantificación de los ensayos de tensión realizados en las muestras antes y después del reproceso, se obtuvo: que el comportamiento de la curva para la muestra Z1 es completamente lineal hasta la carga máxima o punto de falla del material, mientras que para la muestra Z2 comenzó a presentarse una desviación a una carga que está por debajo de la carga máxima de ambas muestras. Lo anterior indica que la muestra Z2 inicia un proceso de deformación, conocido como la cedencia, que evidencia que la muestra tiene cierta capacidad para deformarse antes de fallar.

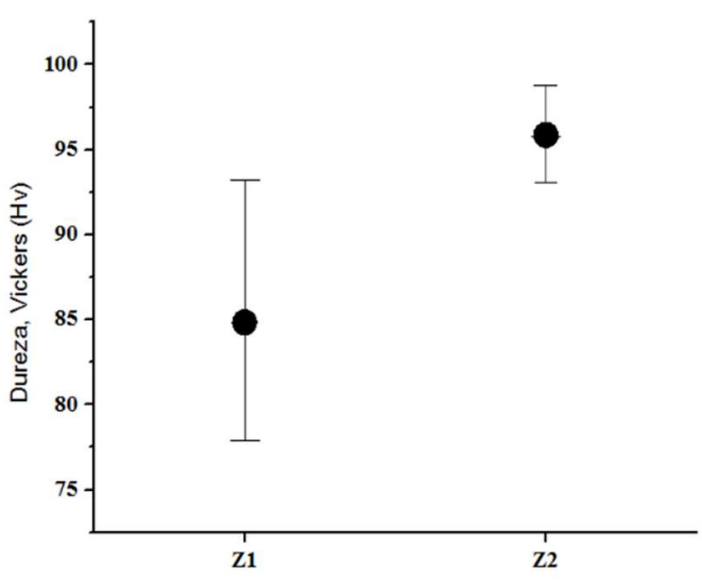

a)

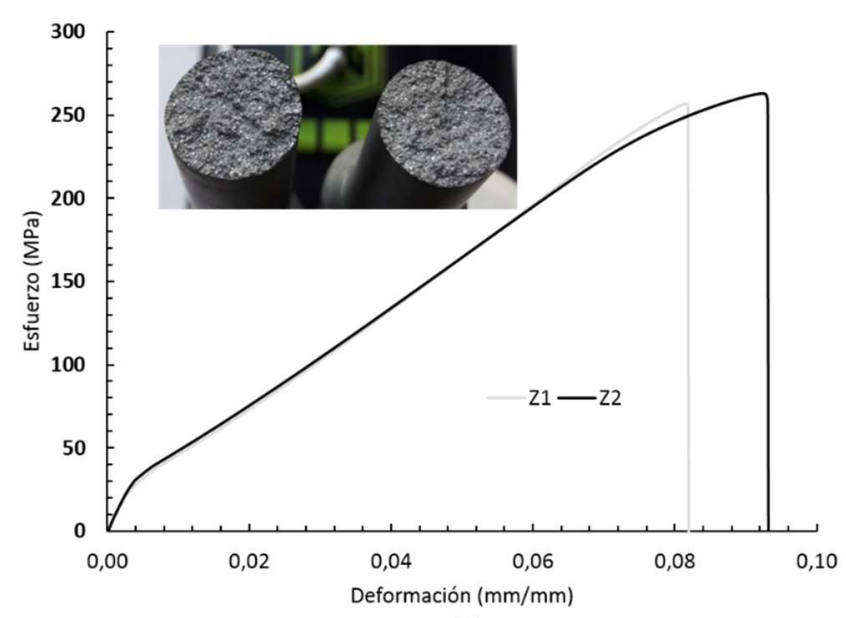

b)

Figura 7. Ensayos mecánicos volumétricos a) ensayos de macrodureza y b) ensayos de tensión

Finalmente, en la imagen de la falla mostrada en la Figura 7.b (parte superior izquierda), se notaron algunas características para ambas muestras, donde se observó una falla frágil debido a que no se presentó deformación plástica notoria antes de la falla. Además, se evidenciaron unas pequeñas marcas de ríos propias de materiales frágiles y estructuras cristalinas hexagonales compactas.

\section{CONCLUSIONES}

Del análisis obtenido al comparar las muestras en estado de entrega con las reprocesadas, se encontró que hay cambios microestructurales y aumento de algunos elementos como el aluminio y el cobre en el material reprocesado, lo que afectó ligeramente las propiedades mecánicas, pero sin diferencias significativas. Sin embargo, este cambio no afectó el proceso de fabricación de conformado volumétrico que se realizó durante el proceso de manufactura de piezas para bisutería.

Después de los tratamientos de refundición y reinyección, se evidenciaron pequeños cambios en la fase eutéctica $(\alpha+\beta)$ y la fase $\beta$, lo que a su vez influyó en las propiedades mecánicas medidas por nanoindentación, pero dichos cambios, no afectaron significativamente las propiedades volumétricas ni la capacidad de deformación del material durante el proceso de inyección. Por todo lo anterior, se concluye que el reciclaje de este material y su reproceso por fusión e inyección es una importante alternativa para la economía del proceso de manufactura de piezas de bisutería sin comprometer las características mecánicas que la pieza requiere. 


\section{REFERENCIAS}

[1] N. S. Giraldo Sánchez, J. D. Valencia, J. M. Meza Meza, y C. A. Isaza Merino, «Estudio de las propiedades tribológicas de una aleación de magnesio AZ31B reforzado con nanotubos de carbono», Rev. CINTEX, vol. 23, n. ${ }^{\circ}$ 2, pp. 54-60, dic. 2018.

[2] E. Mejía, L. Osorno, y J. Ospina, «Microorganismos Hierro-Azufre Oxidantes Una Alternativa Biotecnológica», Rev. CINTEX, vol. 19, pp. 63-77, 2014.

[3] L. V. Rojas Florez y E. A. Hernandez Rincon, «Recuperacion De Retornos De Zamak Niquelado Para La Elaboracion De Aleaciones Con Alto Contenido De Aluminio (Fantaxias).», Thesis, Universidad Industrial de Santander, Escuela De Ingeniería Metalúrgica y ..., 2013.

[4] V. Petrovic, J. Vicente Haro Gonzalez, O. Jordá Ferrando, J. Delgado Gordillo, J. Ramón Blasco Puchades, y L. Portolés Griñan, «Additive layered manufacturing: sectors of industrial application shown through case studies», Int. J. Prod. Res., vol. 49, n. 4, pp. 1061-1079, feb. 2011.

[5] K. Cacua Madero, R. Buitrago-Sierra, E. Pabón Gelves, B. Herrera Múnera, y J. L. Rentería, «Estudio de los factores que afectan la estabilidad de nanofluidos usando un diseño factorial fraccionado», Rev. CINTEX, vol. 22, n. 1, jun. 2017.

[6] H. Pinto y F. J. G. Silva, «Optimisation of Die Casting Process in Zamak Alloys», Procedia Manuf., vol. 11, pp. 517-525, 2017.

[7] A. Azizi y G. G. Haghighi, «Fabrication of ZAMAK 2 alloys by powder metallurgy process», Int. J. Adv. Manuf. Technol., vol. 77, n. ${ }^{\circ}$ 9-12, pp. 2059-2065, abr. 2015.

[8] M. Almomani, M. T. Hayajneh, y M. Draidi, «Tribological investigation of Zamak alloys reinforced with alumina $\left(\mathrm{Al}_{2} \mathrm{O}_{3}\right)$ and fly ash», Part. Sci. Technol., vol. 34, n. ${ }^{\circ}$ 3, pp. 317-323, may 2016.

[9] B. K. Prasad, «Effect of microstructure on the sliding wear performance of a $\mathrm{Zn}-\mathrm{Al}-\mathrm{Ni}$ alloy», Wear, vol. 240, n. ${ }^{\circ} 1-2$, pp. 100-112, may 2000.

[10] J. M. Gobien, R. O. Scattergood, F. E. Goodwin, y C. C. Koch, «Mechanical behavior of bulk ultra-finegrained Zn-Al die-casting alloys», Mater. Sci. Eng. A, vol. 518, n. 1-2, pp. 84-88, ago. 2009.

[11] Z. Wu, S. Sandlöbes, L. Wu, W. Hu, G. Gottstein, y S. Korte-Kerzel, «Mechanical behaviour of Zn-AlCu-Mg alloys: Deformation mechanisms of as-cast microstructures», Mater. Sci. Eng. A, vol. 651, pp. 675687, ene. 2016.

[12] F. Hoyos Gómez, J. D. Betancur Gómez, D. Osorio Patiño, y J. G. Ardila Marín, «Construcción de curvas de factor de concentración de esfuerzos por medio de simulaciones», Rev. CINTEX, vol. 21, n. ${ }^{\circ}$, jun. 2016.

[13] J. A. Patiño-Murillo, J. J. Castro-Maldonado, Y. C. Gutiérrez-Sandoval, J. I. Leal-Santafé, y O. Hurtado-Figueroa, «Estudio del comportamiento de muestras de mortero natural sometidas a esfuerzo de compresión», Lámpsakos, vol. 1, n. ${ }^{\circ}$ 20, pp. 22-28, 2018.

[14] E04 Committee, "Guide for Preparation of Metallographic Specimens», ASTM International.

[15] E28 Committee, «Test Methods for Vickers Hardness and Knoop Hardness of Metallic Materials», ASTM International.

[16] E28 Committee, «Test Methods for Tension Testing of Metallic Materials», ASTM International. 